\title{
IMMUNOREGULATION IN HUMAN SCHISTOSOMIASIS BY IDIOTYPIC INTERACTIONS AND LYMPHOKINE-MEDIATED MECHANISMS
}

\author{
D. G. COLLEY* ${ }^{*}$, J. C. PARRA**, M. A. MONTESANO ${ }^{+}$, M. LIMA** $^{*}$, E. NASCIMENTO ${ }^{+}$, \\ B. L. DOUGHTY ${ }^{++}$, A. GOES ${ }^{* *} /{ }^{++} \&$ G. GAZZINELLI**
}

\begin{abstract}
*VA Medical Center, Vanderbilt Univ., Nashiville TN, USA **Centro de Pesquisas, "Rene Rachou" and Univ. Fed. Minas Gerais, Belo Horizonte, MG, Brasil ${ }^{+}$Univ. Fed, Juiz de Fora, Juiz de Fora, MG, Brasil

${ }^{++}$Texas A \& M Univ., College Station TX, USA
\end{abstract}

Anti-idiotypic (anti-Id) T cells from schistosomiasis patients or former patients proliferate upon exposure to polyclonal or monclonal anti-soluble egg antigen (SEA) antibodies. Chloroquine does not inhibit, the response, which is induced by $F\left(a b^{\prime}\right)_{2}$ (but not soluble Fab) fragments of these antibodies. Purified $T$ cells from former patients require macrophages or exogenous $I L-1$ to respond to anti-SEA Ids and can respond to matrix-bound Fab fragments in the presence of $I L-1$. These anti-Id $T$ cells recognize the Ids directly. Chronic schistosomiasis patients immunoregulate the production of a non-IL-2 lymphokine that stimulates $I L-2$ receptor expression on resting $T$ cells. This regulation is reversed upon chemotherapeutic cure.

Shistosomiasis is a chronic, normally well tolerated infection. While most patients infected with Schistosoma mansoni carry their infections for long periods of time with little overt symptomatology, $5-10 \%$ of the infected population progress to frank hepatosplenic involvement reflective of life-threatening portal hypertension. We and others have reported previously that the chronic, well-tolerated, "intestinal" form of schistosomiasis is associated with the establishment and maintenance of a variety of immunoregulatory mechanisms (D. G. Colley, 1981. p. 1-83. In J. M. Mansfield, Parasitic Diseases, Vol. 1. Marcel Dekker, New York; D. G. Colley, 1987. p. 315-332. In A. A. F. Mahmoud, Balliere's Clinical Tropical Medicine and Communicable Diseases. Vol. 2., W. B. Saunders, Eastbourne, UK). Furthermore, the hepatosplenic condition is often accompanied by a lack of immunoregulation (D. J. Tweardy et al., 1983, Trans. Assoc. Amer. Phys., 96: 392-400; D. G. Colley, et al., 1986, Am. J. Trop. Med. Hyg., 35: 793-802). This report will focus on two immunoregulatory systems we have studied which occur in chronic, intestinal patients and may play roles in the maintenance of a balanced asymptomatic infection.

Idiotypic/anti-idiotypic (Id/anti-Id) interactions have been reported in both murine and

Supported in part by UNDP/World Bank/WHO Special TDR Programme, CNPq, FINEP, AI-11289 and Veterans Administration (USA). human schistosomiasis. In murine schistosomiasis japonica there is a major anti-soluble egg antigenic preparation (SEA)-related, crossreactive idiotype which is central to the immunoregulation of granuloma formation (modulation) (T. F. Kresina \& G. R. Olds, 1986, Inf. Immun., 53: 90-94). Murine schistosomiasis mansoni leads to the development of B cells producing anti-Id antibodies and anti-Id $T$ cells (M. R. Powell \& D. G. Colley, 1985, J. Immunol., 134: 4140-4145; 1987, Cell. Immunol., 104: $377-385$ ), and an anti-Id specific $T$ suppressor factor derived from thymus cells from chronically-infected mice can suppress early granuloma formation upon passive transfer into acutely-infected mice (T. Abe \& D. G. Colley, 1984, J. Immunol, 132: 2084-2088). Id/anti-Id interactions can also influence protective immunity in experimental schistosomiasis ( $\mathrm{J}$. M. Grzych et al., 1985, Nature, 316: 74-76; S. M. Phillips et al., 1986, J. Immunol., 137: 2339-2347).

We have recently demonstrated the presence of anti-Id specific $T$ lymphocytes in the peripheral blood mononuclear cells (PBMN) of schistosomiasis patients and former patients. These cells are detected by their proliferation in culture when exposed to anti-SEA antibodies from intestinal patients (M. S. Lima et al., 1986, J. Clin. Invest., 78: 983-988). These anti-Id $\mathrm{T}$ cell responses require the presence of adherent (Adh) cells which are predominantly macrophages. To better define the role of these 
Adh cells in this response, and determine the nature of the stimulation of $T$ cells by anti-SEA antibodies, we have studied the interaction of PBMN or $T$ cells from former patients (cured of their schistosomiasis more than 5 years previously) and these anti-SEA antibodies.

Anti-SEA antibodies (Ids) were purified by immunoaffinity chromatography over SEASepharose 4B, and added to cell cultures containing 150,000 cells in flat-bottom microwell tissue culture plates and $200 \mu$ of complete medium (RPMI 1640, 5\% normal human serum, penicillin-streptomycin, $1 \%$ L-glutamine) (Lima et al., J. Clin. Invest., 78: 983-988). In some cases human monoclonal anti-SEA antibodies were used in these cultures. These antibodies were produced by hybridomas prepared from patients' PBMN (A. Goes \& B. L. Doughty, manuscript in preparation). Data from experiments with the PBMN from 4 patients cultured in the presence of polyclonal anti-SEA Ids or 2 different human monoclonal anti-SEA antibodies (E5 and A7) are presented in the Table.

TABLE

PBMN responses ( $\mathrm{E}-\mathrm{C}$ values in $\mathrm{CPM}$ ) to a polyclonal anti-SEA eluate or 2 human anti-SEA monoclonal antibodies

\begin{tabular}{crrr}
\hline & & \multicolumn{2}{c}{ Anti-SEA monoclonals } \\
\cline { 3 - 4 } Patients & anti-SEA eluate & \multicolumn{1}{c}{ E5 } & \multicolumn{1}{c}{ A7 } \\
\hline 1 & 20730 & 33857 & 14 \\
2 & 16562 & 34888 & 299 \\
3 & 17845 & 43167 & 485 \\
4 & 31100 & 315 & 683 \\
\hline
\end{tabular}

It is clear from the data in the Table that the human anti-SEA monoclonal antibody E5 is capable of stimulating proliferation of some patients' PBMN, as measured by incorporation of tritiated thymidine. None of the patients tested thus far have had cells responsive to monoclonal A7. In the exemples shown in the Table monoclonal E5 actually stimulated higher degrees of proliferation than did the polyclonal Ids. Because the preparations were all used at $40 \mu \mathrm{g} / \mathrm{ml}$ final protein concentration in the cultures, it is possible that this could be due to a higher effective concentration of a given dominant, stimulatory Id being achieved by use of the monoclonal.

The effect of chloroquine treatment on the response of former patients' PBMN to SEA was, as expected (K. Ziegler \& E. R. Unanue, 1982, Proc. Natl. Acad. Sci., USA, 79: 175), i.e. proliferation was inhibited whether chloroquine was present throughout the culture $(25 \mu \mathrm{M})$ or only during a pre-pulsing phase $\left(1 \mathrm{hr} / 37^{\circ} \mathrm{C} /\right.$ $400 \mathrm{M}$ ). However, when these conditions were used in parallel in Id-exposed cultures, stimulation was still observed (J. C. Parra et al., 1988, J. Immunol., 140: 2401-2405). This indicates that "antigen processing" is not required in this system for Id-mediated stimulation of PBMN.

PBMN from former patients were separated into non-adherent (NA, T cell-enriched) cells and Adh cells, the Adh cells fixed with $0.15 \%$ paraformaldehyde for 2 minutes and washed. The fixed cells were used (pulsed and cocultured) immediately after fixation and washing. They were not first cultured overnight. In the experiment shown in Fig. 1, unfixed (living) or fixed, washed Adh cells were pulsed (with either medium only, SEA, or anti-SEA Ids) and co-cultured with autologous NA cells (150,000 of each in this experiment). The results demonstrate that autologous A cells which are fixed and then pulsed with SEA do not stimulate NA cells. Unfixed, SEApulsed A cells did stimulate NA cells. However, both fixed and unfixed autologous Adh cells pulsed with anti-SEA Ids stimulated NA cells to proliferate (Fig. 2). Other experiments with other former patients' cells which used a different fixation (0.01\% paraformaldehyde/ 1 minute) and the addition of 25,000 Adh cells to $150,000 \mathrm{NA}$ cells produced very similar findings to those presented in Figure 1 (data not shown). The finding that fixed Adh cells can provide the role of Adh cells in Id-stimulated, but not SEA-stimulated cultures is consistent with the chloroquine studies described above. Because fixed macrophages can "leak" IL-1 for several hours and express membranebound IL-1 (C. T. Weaver \& E. R. Unanue, 1986, J. Immunol., 137: 3868-3873), these studies do not address the possibility that the Adh cells are required for the production of IL-1. It seems clear that Adh cell "processing" is not required for Id stimulation. 


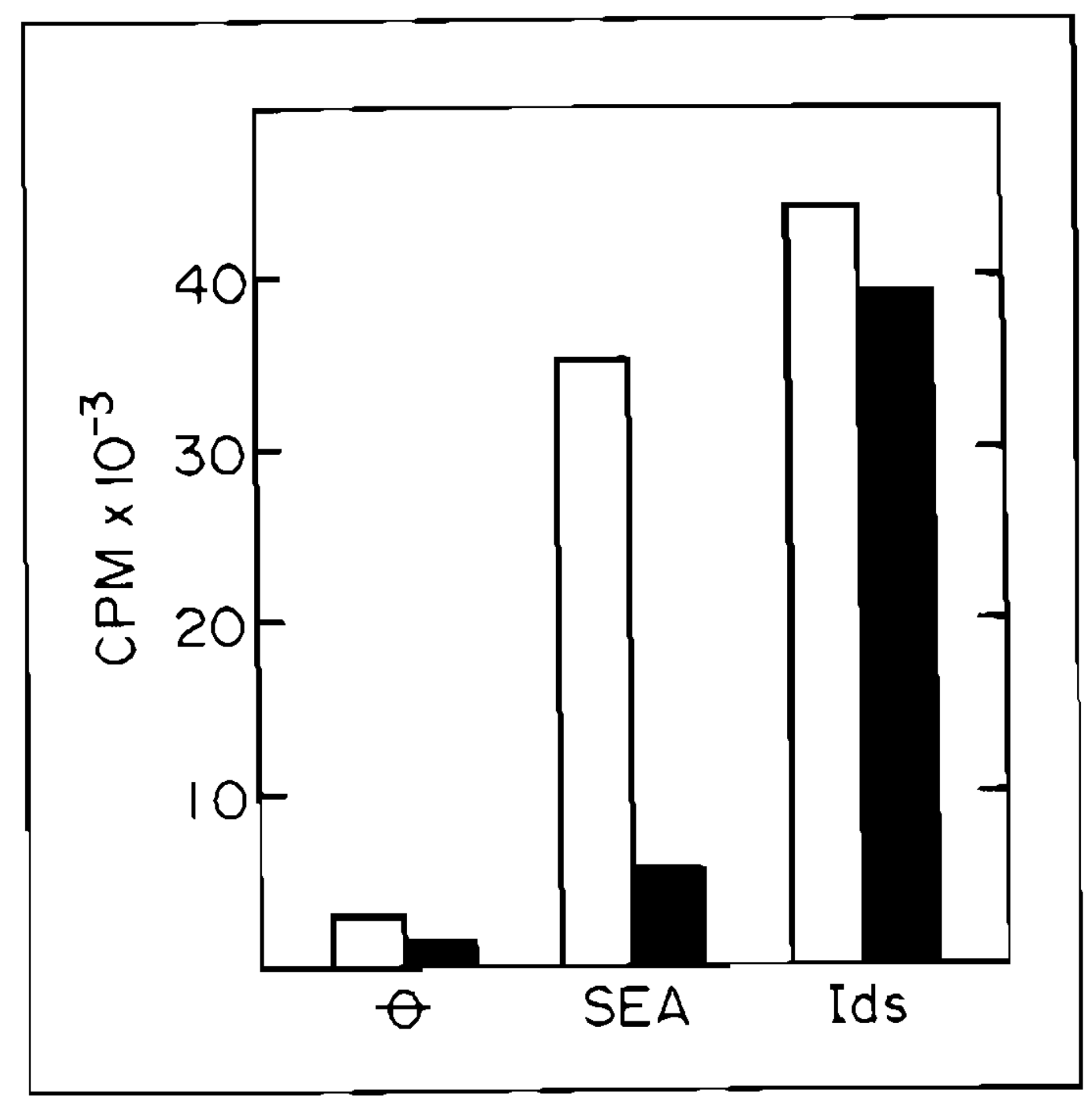

Fig. 1: Adh cells from a former patient were fixed for 2 minutes with $0.15 \%$ paraformaldehyde and washed multiple times. Fixed ( $\square$ and unfixed ( $\square$ ) Adh cells $(150,000)$ were co-cultured with 150,000 autologous NA cells in the presence of either medium (O), SEA $(25 \mu \mathrm{g} / \mathrm{ml})$ or anti-SEA Ids $(30 \mu \mathrm{g} / \mathrm{ml})$.

As described previously (Lima et al., J. Clin. Invest., 78: 983-988; Parra et al., 1988, J. Immunol., 140: 2401-1405), $\mathrm{F}(\mathrm{ab})_{2}$ fragments, but not Fc or Fab fragments of the stimulatory Ids retain their stimulatory activity for PBMN of former patients. However, if Fab fragments prepared from stimulatory anti-SEA antibodies are coupled to Sepharose 4B and incubated with NA cells from a former patient in the presence of purified human IL-1 (5 half-maximum units/ml culture; Cistron, Pine Brook, NJ, USA) they can induce proliferation (Fig. 2). Thus, although Fab fragments are nonstimulatory, Fab fragments which can provide a cross-linked signal due to their presentation on a matrix are stimulatory in the presence of exogenous IL-1. Polyclonal rabbit anti-IL-1 inhibits this supportive role of exogenously added IL-1 (Fig. 2) (J. C. Parra et al., 1988, J. Immunol., 140: 2401-2405).

The data presented above demonstrate that in this system SEA and anti-SEA Ids are recognized in different and distinct ways. Lymphocyte stimulation by SEA requires processing and presentation, while anti-SEA Ids can stimulate appropriate $\mathrm{T}$ cells directly, without a requirement for processing or MHC-restricted recognition. In the absence of Adh cells, Idstimulated proliferation does depend on an exogenous source of IL-1.

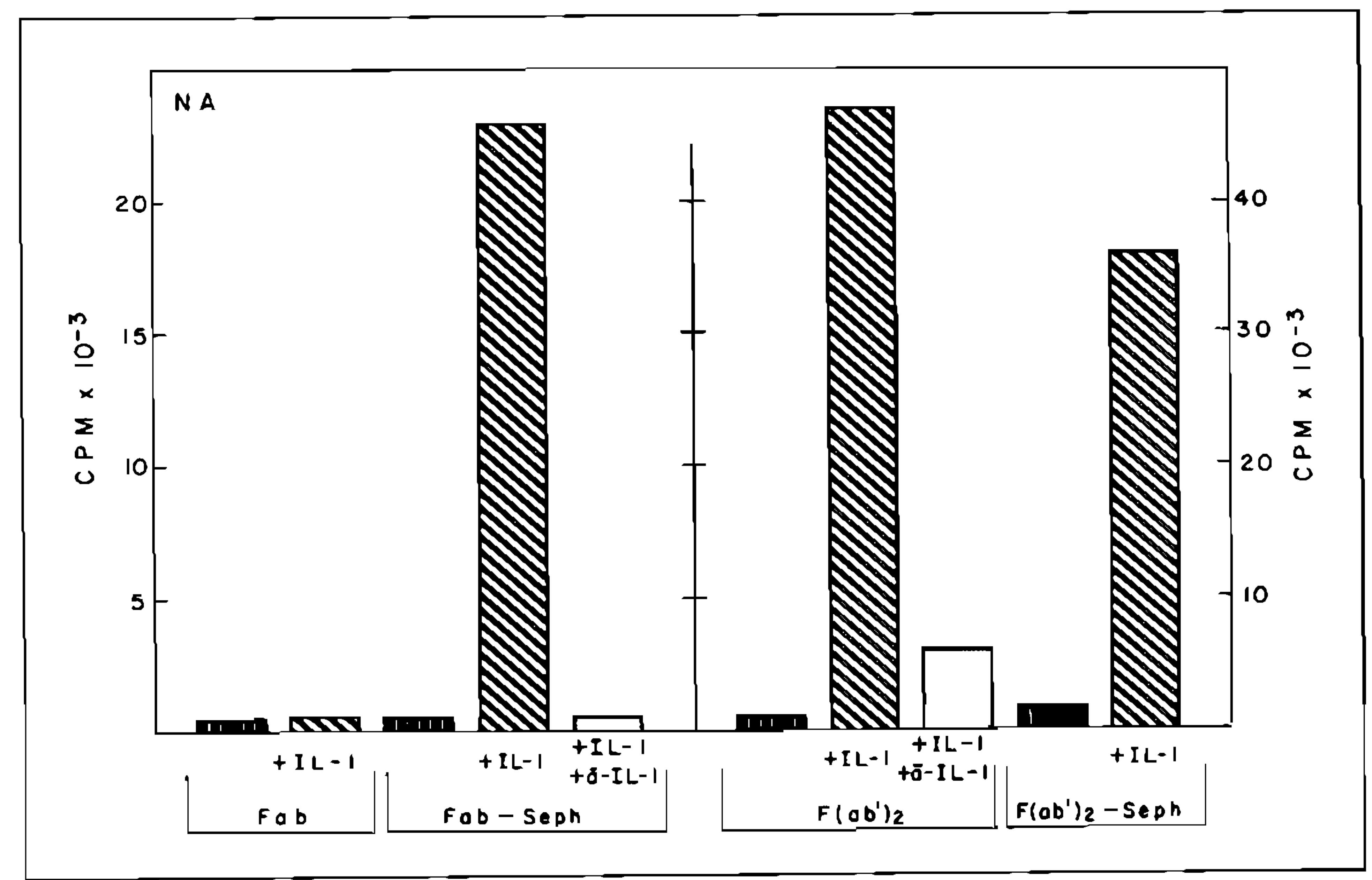

Fig. 2: NA cells from a former patient were cultured (6 days) with anti-SEA F $\left(\mathrm{ab}^{\prime}\right)_{2}$ or Fab Ids in either soluble or matrix (Sepharose 4B, Seph) bound form, in the presence or absence of exogenous IL-1. IL-1 containing cultures were maintained in the presence or absence of rabbit anti-IL-1 serum. 
The presence of anti-SEA Id specific $T$ cells in the PBMN cells of patients and former patients provides an opportunity for the generation of a variety of immunoregulatory phenomena. Stimulation of these cells is seen to lead to their ability to suppress the formation of anti-SEA focused granulomas in vitro (A. Goes et al., manuscript in preparation). In the murine model of schistosomiasis mansoni there is evidence for anti-Id $\mathrm{T}$ cell involvement in granuloma modulation in vivo, as manifested by the anti-idiotypic nature of a $\mathrm{T}$ suppressor factor derived from thymocytes or spleen/ lymph node cells from mice with chronic infections (T. Abe \& D. G. Colley, 1984, J. Immunol., 132: 2084-2088). The regulation of granuloma formation stimulated by an immunodominant, cross-reactive idiotype has also been shown in murine schistosomiasis japonica infections (T. Kresina \& G. R. Olds, 1986, Infect. Immun., 53: 90-94). It is possible that these idiotypic-anti-idiotypic influences may begin prior to birth in persons born of mothers with active schistosomiasis during their pregnancies. Many of these pergnant females will have high titers of anti-SEA antibodies, some of which may bear immunodominant, crossreactive Ids. When their antibodies cross the placenta to the fetus they could influence the generation and/or survival of developing lymphocytes and therefore the repertoires subsequently expressed in the offspring.

The other form of immune regulatory interactions to be discussed concerns an obser. vation by Gazzinelli ct al. (1983, J. Immunol., 130: 2891-2895) that exposure of former patients' PBMN cells to schistosomal worm antigenic preparation (SWAP) results in the production and release of an activity in the supernatant fluids which, over 6 days of culture, leads to detectable proliferation of resting PBMN from normal subjects. We referred to this acitivity as mitogenic factor (MF). PBMN from approximately $75 \%$ of patients with active, intestinal schistosomiasis mansoni do not produce MF activity upon SWAP stimulation (Gazzinelli et al., 1983, J. Immunol., 130:2891. 2895). We believe this lack of production reflects an immunoregulation of the ability to make this factor, and this regulation is lost upon chemotherapeutic cure. The moiety(ies) responsible for MF acitivity have been generally characterized as glycoprotein in nature, and MF activity is produced by former patients' $\mathrm{T}$ lymphocytes, not $\mathrm{B}$ cells or macro- phages (M. A. Montesano, M. S. Thesis, UFMG, Belo Horizonte, MG; D. G. Colley et al., 1987, p. 333-347, in A. J. Maclnnis Molecular Paradigms for Eradicating Helminth Parasites, Alan R. Liss, New York).

There is now evidence that this immunoregulated function may be involved in the responsiveness of $\mathrm{T}$ lymphocytes. If MF-containing supernatant fluids are depleted of IL-2 acitivity by immunoaffinity absorptions, the overall mitogenic acitivity defined by the proliferative MF assay is lost in parallel (data not shown). However, previously MF-containing supernatant fluids depleted of IL-2 do still have an effect on resting normal PBMN over the course of a 6 day culture. Although these IL-2-depleted fluids lack proliferative activity on either IL-2dependent CTLL-2 cells or resting normal PBMN, they stimulate resting PBMN to express IL-2 receptors (IL-2R) and respond to exogenous IL-2. For example, using anti-IL-2R immunofluorescence we found that after 2 and 6 days of culture, control cells were $2-4 \%$ positive and $2-5 \%$ positive for IL-2R expression, respectively. However, if resting PBMN were exposed to either unabsorbed or anti-IL-2absorbed culture fluids, by day 2 they were $4-6 \%$ positive and on day 6 they were $11.15 \%$ positive. This lymphokine-stimulated expression of $1 \mathrm{~L}-2 \mathrm{R}$ by resting PBMN occurred whether the fluids did or did not contain detectable levels of IL-2 (data not shown). Parallel studies revealed that cultures that contain cells which have been stimulated to express surface IL-2R also become responsive (proliferate) in response to exogenous IL-2 (data not shown).

We conjecture that the immunoregulated production of this IL-2R-inducing factor (IL-2R-IF) during chronic intestinal schistosomiasis may result in a diminished generalized responsiveness upon specific stimulation by schistosomal antigens. Furthermore, the data may provide an indication of the critical events which occur between antigen-cell interaction and the initial expression of cell surface IL-2R. It is known that IL-2 itself can regulate the expression of $I L-2 R$ on cells which already express elevated numbers of IL-2R (G. H. Reem \& N.-N. Yeh, 1984, Science, 225: 429-430; K. A. Smith \& D. A. Cantrell, 1985, Proc. Natl. Acad. Sci., USA, 82: 864 . 868 ), but it remains unclear how antigen or phytomitogen activation induces the initial 
elevation in IL-2R surface expression (J. D. somiasis it may be possible to exploit this Ashwell et al., 1986. J. Immunol., 137:2572- system to learn more about this control, 2578). If this is the process that is immuno- thereby assisting in an undrestanding of the regulated in chronic, immunoregulated schisto- nature of immunoregulation in this infection. 